\title{
BILINEAR MULTIPLIERS AND TRANSFERENCE
}

\author{
OSCAR BLASCO
}

Received 26 April 2004 and in revised form 29 October 2004

We give de Leeuw-type transference theorems for bilinear multipliers. In particular, it is shown that bilinear multipliers arising from regulated functions $m(\xi, \eta)$ in $\mathbb{R} \times \mathbb{R}$ can be transferred to bilinear multipliers acting on $\mathbb{T} \times \mathbb{T}$ and $\mathbb{Z} \times \mathbb{Z}$. The results follow from the description of bilinear multipliers on the discrete real line acting on $L^{p}$-spaces.

\section{Introduction}

Let $\left(p_{1}, p_{2}, p_{3}\right)$ be such that $0<p_{1}, p_{2}, p_{3} \leq \infty, 1 / p_{1}+1 / p_{2}=1 / p_{3}$ and let $m(\xi, \eta)$ be a bounded measurable function on $\mathbb{R}^{2}$. It is said to be a bilinear $\left(p_{1}, p_{2}\right)$-multiplier on $\mathbb{R} \times \mathbb{R}$ if

$$
\mathscr{C}_{m}(f, g)(x)=\int_{\mathbb{R}^{2}} \hat{f}(\xi) \hat{g}(\eta) m(\xi, \eta) e^{2 \pi i x(\xi+\eta)} d \xi d \eta
$$

(defined for Schwarzt test functions $f$ and $g$ in $\mathscr{S}$ ) extends to a bounded bilinear operator from $L^{p_{1}}(\mathbb{R}) \times L^{p_{2}}(\mathbb{R})$ into $L^{p_{3}}(\mathbb{R})$.

The theory of these multipliers has been tremendously developed after the results proved by Lacey and Thiele (see $[16,18,17])$ which establish that $m(\xi, \nu)=\operatorname{sign}(\xi+\alpha \nu)$ is a $\left(p_{1}, p_{2}\right)$-multiplier for each triple $\left(p_{1}, p_{2}, p_{3}\right)$ such that $1<p_{1}, p_{2} \leq \infty, p_{3}>2 / 3$, and each $\alpha \in \mathbb{R} \backslash\{0,1\}$.

The study of such multipliers was started by Coifman and Meyer (see $[3,4,19])$ for smooth symbols and new results for nonsmooth symbols, extending the ones given by the bilinear Hilbert transform, have been achieved by Gilbert and Nahmod (see $[8,9,10]$ ) and also by Muscalu et al. (see [20]).

We refer the reader also to $[7,12,11,15]$ for new results on bilinear multipliers and related topics.

In a recent paper (see [7]), Fan and Sato have shown certain de Leeuw-type theorems for transferring multilinear operators on Lebesgue and Hardy spaces from $\mathbb{R}^{n}$ to $\mathbb{T}^{n}$. Here we will consider bilinear multipliers on Lebesgue spaces $L^{p}(\mathbb{R})$ and get a characterization which allows us to transfer not only to the bilinear multipliers on $\mathbb{T}$ but also on $\mathbb{Z}$. Our approach will follow closely the ideas in the original paper by de Leeuw (see [6]) and will 
provide an alternative proof of some results in [7], whose proof follows, in the multilinear case, the approach used by Stein and Weiss (see [21, page 260]).

We start by setting up natural analogous versions of bilinear multipliers in the periodic and discrete cases. Let $m=\left(m_{k, k^{\prime}}\right)$ be a bounded sequence and let $\tilde{m}$ be a periodic function on $\mathbb{\mathbb { V }} \times \mathbb{T}$. Define for $\theta \in[-1 / 2,1 / 2]$,

$$
\mathscr{P}_{m}(f, g)(\theta)=\sum_{k \in \mathbb{Z}} \sum_{k^{\prime} \in \mathbb{Z}} \hat{f}(k) \hat{g}\left(k^{\prime}\right) m_{k, k^{\prime}} e^{2 \pi i \theta\left(k+k^{\prime}\right)}
$$

for functions $f, g$ defined on $\mathbb{T}$, and for $k \in \mathbb{Z}$,

$$
\mathscr{D}_{\tilde{m}}(a, b)(k)=\int_{-1 / 2}^{1 / 2} \int_{-1 / 2}^{1 / 2} P(t) Q(s) \tilde{m}(t, s) e^{2 \pi i k(t+s)} d t d s
$$

for sequences $a=(a(n))_{n \in \mathbb{Z}}$ and $b=(b(n))_{n \in \mathbb{Z}}$, where $P(t)=\sum_{n \in \mathbb{Z}} a(n) e^{2 \pi i n t}$ and $Q(t)=$ $\sum_{n \in \mathbb{Z}} b(n) e^{2 \pi i n t}$.

Now we say that $m$ (resp., $\tilde{m})$ is a bilinear $\left(p_{1}, p_{2}\right)$-multiplier on $\mathbb{Z} \times \mathbb{Z}($ resp., $\mathbb{T} \times \mathbb{T}$ ) if $\mathscr{P}_{m}$ (resp., $\left.\mathscr{D}_{\tilde{m}}\right)$ defines a bounded bilinear operator from $L^{p_{1}}(\mathbb{T}) \times L^{p_{2}}(\mathbb{T})$ into $L^{p_{3}}(\mathbb{T})$ (resp., $\ell^{p_{1}}(\mathbb{Z}) \times \ell^{p_{2}}(\mathbb{Z})$ into $\left.\ell^{p_{3}}(\mathbb{Z})\right)$, where $1 / p_{1}+1 / p_{2}=1 / p_{3}$.

Of course we can see these three cases as instances of the general bilinear multiplier acting on different groups. Let $G$ be a locally compact abelian group and $\hat{G}$ its dual group with Haar measure $\mu$. Let $1 \leq p_{1}, p_{2} \leq \infty$ and let $m$ be a bounded measurable function on $\hat{G} \times \hat{G}$. We say that $m$ is a $\left(p_{1}, p_{2}\right)$-multiplier on $\hat{G} \times \hat{G}$ if the operator

$$
T_{m}(f, g)(x)=\int_{\widehat{G}} \int_{\widehat{G}} \mathscr{F} f\left(\gamma_{1}\right) \mathscr{F} g\left(\gamma_{2}\right) m\left(\gamma_{1}, \gamma_{2}\right) \gamma_{1}(-x) \gamma_{2}(-x) d \mu\left(\gamma_{1}\right) d \mu\left(\gamma_{2}\right)
$$

(defined for simple functions $f$ and $g$ ) extends to a bounded bilinear operator from $L^{p_{1}}(G) \times L^{p_{2}}(G)$ to $L^{p_{3}}(G)$, where $1 / p_{1}+1 / p_{2}=1 / p_{3}$. The reader is referred to [14] for the general theory in the linear case.

The first transference results on linear multipliers were given by de Leeuw (see [6]). He showed, among other things, that if $m$ is regulated (all its points are Lebesgue points) and $m$ is a $p$-multiplier on $\mathbb{R}$, then $(m(\varepsilon k))_{k}$ is a uniformly bounded $p$-multiplier for all $\varepsilon>0$ on $\mathbb{Z}$ (see [21, page 264] for the converse of this result for continuous multipliers). Transference results of similar nature are presented in [1].

A general transference method was considered by [5] (see also the generalization given by [13]), but we will not consider these approaches in our bilinear generalization in the paper.

In [7], the multilinear version of the continuous result was shown, namely that for any continuous function $m(\xi, \eta)$, one has that $m$ is a $\left(p_{1}, p_{2}\right)$-multiplier on $\mathbb{R} \times \mathbb{R}$ if and only if $m\left(\varepsilon k, \varepsilon k^{\prime}\right)_{k, k^{\prime}}$ is a uniformly bounded multiplier on $\mathbb{Z} \times \mathbb{Z}$ for $\varepsilon>0$. An extension of the result to Lorentz spaces was achieved in [2]. 
We will first characterize the boundedness of bilinear multipliers on $\mathbb{R} \times \mathbb{R}$ by the existence of a constant $K$ such that

$$
\left|\sum_{t \in \mathbb{R}} \sum_{s \in \mathbb{R}} m(t, s) \mu(\{t\}) \nu(\{s\}) \lambda(\{t+s\})\right| \leq K\|\hat{\mu}\|_{B_{p_{1}}}\|\hat{\nu}\|_{B_{p_{2}}}\|\hat{\lambda}\|_{B_{p_{3}^{\prime}}}
$$

for all measures $\mu, \nu, \lambda$ of finite supports.

This allows us to transfer from the continuous $\mathscr{C}_{m}$ to the discrete case $\mathscr{D}_{\tilde{m}}$ recovering some of the Fan-Sato results in [7].

We also obtain the transference from the continuous case $\mathscr{C}_{m}$ to the periodic case $\mathscr{P}_{m}$. Our main result establishes that $m$ is a $\left(p_{1}, p_{2}\right)$-multiplier on $\mathbb{R} \times \mathbb{R}$ if and only if $D_{\varepsilon} m=m_{\varepsilon \cdot, \varepsilon} \cdot \chi_{[-1 / 2,1 / 2] \times[-1 / 2,1 / 2]}$ (extended by periodicity) are uniformly bounded $\left(p_{1}, p_{2}\right)$ multipliers on $\mathbb{T} \times \mathbb{T}$.

The reader should be aware that the results of the paper can be stated for multilinear multipliers, with the condition $1 / p=\sum_{i=1}^{n}\left(1 / p_{i}\right)$, by considering the corresponding multilinear notions, for instance, for $m\left(\xi_{1}, \ldots, \xi_{n}\right)$, one has

$$
\mathscr{b}_{m}\left(f_{1}, \ldots, f_{n}\right)(x)=\int_{\mathbb{R}^{n}} \hat{f}_{1}\left(\xi_{1}\right) \cdots \hat{f}_{n}\left(\xi_{n}\right) m\left(\xi_{1}, \ldots, \xi_{n}\right) e^{2 \pi i x\left(\xi_{1}+\cdots+\xi_{n}\right)} d \xi_{1} \cdots d \xi_{n}
$$

and similar modifications for $\mathscr{P}_{m}$ and $\mathscr{D}_{\tilde{m}}$. We simply do the bilinear case for the sake of simplicity.

Throughout the paper, $1 \leq p_{1}, p_{2}, p_{3} \leq \infty$ and $1 / p_{3}=1 / p_{1}+1 / p_{2}$. For a given finite Borel measure $\mu$ on $\mathbb{R}$, we write $\hat{\mu}(\xi)=\int_{\mathbb{R}} e^{-2 \pi i \xi t} d \mu(t)$ and, for an almost periodic function $g$, we denote $\|g\|_{B_{p}}=\lim _{T \rightarrow \infty}\left((1 / 2 T) \int_{-T}^{T}|g(t)|^{p} d t\right)^{1 / p}$. We will use the notations $D_{\varepsilon} m(x, y)=m(\varepsilon x, \varepsilon y)$ and $\phi_{\varepsilon}(x)=(1 / \varepsilon) \phi(x / \varepsilon)$.

\section{Bilinear multipliers on $\mathbb{R} \times \mathbb{R}$}

We start by reformulating the condition of $\left(p_{1}, p_{2}\right)$-multiplier on $\mathbb{R} \times \mathbb{R}$ using duality. The proof is straightforward and is left to the reader.

Lemma 2.1. Let $m(\xi, \eta)$ be a bounded measurable function on $\mathbb{R} \times \mathbb{R}$. Then $m$ is a $\left(p_{1}, p_{2}\right)$ multiplier on $\mathbb{R} \times \mathbb{R}$ if and only if there exists a constant $K$ so that

$$
\left|\int_{\mathbb{R}^{2}} \phi(\xi) \psi(\eta) \nu(\xi+\eta) m(\xi, \eta) d \xi d \eta\right| \leq K\|\hat{\phi}\|_{p_{1}}\|\hat{\psi}\|_{p_{2}}\|\hat{\nu}\|_{p_{3}^{\prime}}
$$

for all $\phi, \psi, \nu \in \mathscr{S}$.

Now we present some behavior of multipliers on $\mathbb{R} \times \mathbb{R}$ with respect to convolution and dilation operators to be used later on.

Lemma 2.2. Let $m(\xi, \eta)$ be a bounded measurable function on $\mathbb{R} \times \mathbb{R}$. If $\Phi \in L^{1}\left(\mathbb{R}^{2}\right)$ and $m$ is a $\left(p_{1}, p_{2}\right)$-multiplier on $\mathbb{R} \times \mathbb{R}$, then $\Phi * m$ is a $\left(p_{1}, p_{2}\right)$-multiplier on $\mathbb{R} \times \mathbb{R}$ and $\left\|\mathscr{C}_{\Phi * m}\right\| \leq\|\Phi\|_{1}\left\|\mathscr{C}_{m}\right\|$, where $\left\|\mathscr{C}_{m}\right\|$ stands for the norm of the corresponding bilinear map from $L^{p_{1}}(\mathbb{R}) \times L^{p_{2}}(\mathbb{R})$ into $L^{p_{3}}(\mathbb{R})$. 
Proof. Let $f_{s}(x)=f(x+s)$ for any $s \in \mathbb{R}$ and function $f$. Then for any $s, t \in \mathbb{R}$ and $\phi, \psi, v \in$ $\mathscr{S}$ with $\|\hat{\phi}\|_{p_{1}}=\|\hat{\psi}\|_{p_{2}}=\|\hat{\nu}\|_{p_{3}^{\prime}}=1$, we have

$$
\left|\int_{\mathbb{R}^{2}} \phi_{s}(\xi) \psi_{t}(\eta) \nu_{t+s}(\xi+\eta) m(\xi, \eta) d \xi d \eta\right| \leq K
$$

Now

$$
\begin{aligned}
\int_{\mathbb{R}^{2}} \phi(\xi) \psi(\eta) \nu(\xi+\eta) \Phi * m(\xi, \eta) d \xi d \eta \\
\quad=\int_{\mathbb{R}^{2}} \phi(\xi) \psi(\eta) \nu(\xi+\eta)\left(\int_{\mathbb{R}^{2}} m(\xi-s, \eta-t) \Phi(s, t) d s d t\right) d \xi d \eta \\
\quad=\int_{\mathbb{R}^{2}} \int_{\mathbb{R}^{2}} \phi(\xi+s) \psi(\eta+t) \nu(\xi+\eta+s+t) m(\xi, \eta) \Phi(s, t) d \xi d \eta d s d t
\end{aligned}
$$

And the result follows by Lemma 2.1.

Lemma 2.3. Let $\varepsilon>0$ and $m(\xi, \eta)$ be $a\left(p_{1}, p_{2}\right)$-multiplier on $\mathbb{R} \times \mathbb{R}$. Then $m(\varepsilon \xi$, $\varepsilon \eta)$ is also $a\left(p_{1}, p_{2}\right)$-multiplier on $\mathbb{R} \times \mathbb{R}$ and $\left\|\mathscr{C}_{m\left(\varepsilon^{\cdot}, \varepsilon^{\cdot}\right)}\right\| \leq\left\|\mathscr{C}_{m}\right\|$.

Proof. For $\phi, \psi, v \in \mathscr{S}$ and $\|\hat{\phi}\|_{p_{1}}=\|\hat{\psi}\|_{p_{2}}=\|\hat{\nu}\|_{p_{3}^{\prime}}=1$, we have

$$
\begin{aligned}
\int_{\mathbb{R}^{2}} \phi(\xi) \psi(\eta) \nu(\xi+\eta) m(\varepsilon \xi, \varepsilon \eta) d \xi d \eta \\
\quad=\int_{\mathbb{R}^{2}} \frac{1}{\varepsilon^{1 / p_{1}^{\prime}}} \phi\left(\frac{\xi}{\varepsilon}\right) \frac{1}{\varepsilon^{1 / p_{2}^{\prime}}} \psi\left(\frac{\eta}{\varepsilon}\right) \frac{1}{\varepsilon^{1 / p_{3}}} \nu\left(\frac{\xi+\eta}{\varepsilon}\right) m(\xi, \eta) d \xi d \eta
\end{aligned}
$$

The proof is finished invoking Lemma 2.1 again.

Theorem 2.4. Let $m(\xi, \eta)$ be a bounded continuous function on $\mathbb{R} \times \mathbb{R}$. The following are equivalent:

(i) $m$ is a $\left(p_{1}, p_{2}\right)$-multiplier on $\mathbb{R} \times \mathbb{R}$;

(ii) there exists a constant $K$ so that

$$
\left|\sum_{t \in \mathbb{R}} \sum_{s \in \mathbb{R}} m(t, s) \mu(\{t\}) \nu(\{s\}) \lambda(\{t+s\})\right| \leq K\|\hat{\mu}\|_{B_{p_{1}}}\|\hat{\nu}\|_{B_{p_{2}}}\|\hat{\lambda}\|_{B_{p_{3}^{\prime}}}
$$

for all measures $\mu, \nu, \lambda$ supported on a finite number of points.

Proof. (i) $\Rightarrow$ (ii). Assume that $m$ is a $\left(p_{1}, p_{2}\right)$-multiplier on $\mathbb{R} \times \mathbb{R}$. Denote by $\phi$ the Gaussian function $\phi(x)=e^{-x^{2} / 2}$. Then for any $\alpha>0$ and $a \in \mathbb{R}$,

$$
\left(\frac{1}{\varepsilon}\right)^{\alpha} \phi^{\alpha}\left(\frac{\xi-a}{\varepsilon}\right)=\delta_{a} *\left(\phi_{\varepsilon}\right)^{\alpha}(\xi) .
$$


Now choose $0<\alpha, \beta, \gamma$ such that $\alpha+\beta+\gamma=2$, and $\mu=\delta_{a}, \nu=\delta_{b}$, and $\lambda=\delta_{c}$ for $a, b, c \in \mathbb{R}$. It is easily checked that

$$
\begin{aligned}
\int_{\mathbb{R}^{2}} \frac{1}{\varepsilon^{2}} \phi^{\alpha}\left(\frac{\xi-a}{\varepsilon}\right) \phi^{\beta}\left(\frac{\eta-b}{\varepsilon}\right) \phi^{\gamma}\left(\frac{\xi+\eta-c}{\varepsilon}\right) m(\xi, \eta) d \xi d \eta \\
\quad=\int_{\mathbb{R}^{2}} \phi^{\alpha}(\xi) \phi^{\beta}(\eta) \phi^{\gamma}\left(\xi+\eta+\frac{a+b-c}{\varepsilon}\right) m(a+\varepsilon \xi, b+\varepsilon \eta) d \xi d \eta \\
=\int_{\mathbb{R}^{2}} \mu *\left(\phi_{\varepsilon}\right)^{\alpha}(\xi) \nu *\left(\phi_{\varepsilon}\right)^{\beta}(\eta) \lambda *\left(\phi_{\varepsilon}\right)^{\gamma}(\xi+\eta) m(\xi, \eta) d \xi d \eta
\end{aligned}
$$

Since

$$
\begin{gathered}
\lim _{\varepsilon \rightarrow 0} \phi^{\alpha}(\xi) \phi^{\beta}(\eta) \phi^{\gamma}\left(\xi+\eta+\frac{a+b-c}{\varepsilon}\right) m(a+\varepsilon \xi, b+\varepsilon \eta) \\
=\delta_{c}(a+b) \phi^{\alpha}(\xi) \phi^{\beta}(\eta) \phi^{\gamma}(\xi+\eta) m(a, b),
\end{gathered}
$$

the Lebesgue convergence theorem implies that

$$
\begin{aligned}
\lim _{\varepsilon \rightarrow 0} \int_{\mathbb{R}^{2}} & \frac{1}{\varepsilon^{2}} \phi^{\alpha}\left(\frac{\xi-a}{\varepsilon}\right) \phi^{\beta}\left(\frac{\eta-b}{\varepsilon}\right) \phi^{\gamma}\left(\frac{\xi+\eta-c}{\varepsilon}\right) m(\xi, \eta) d \xi d \eta \\
& =C m(a, b) \delta_{c}(a+b)=C m(a, b) \mu(\{a\}) \nu(\{b\}) \lambda(\{a+b\}),
\end{aligned}
$$

where $C=\int_{\mathbb{R}^{2}} \phi^{\alpha}(\xi) \phi^{\beta}(\eta) \phi^{\gamma}(\xi+\eta) d \xi d \eta$.

Therefore we have

$$
\begin{gathered}
\lim _{\varepsilon \rightarrow 0} \int_{\mathbb{R}^{2}} \mu *\left(\phi_{\varepsilon}\right)^{\alpha}(\xi) \nu *\left(\phi_{\varepsilon}\right)^{\beta}(\eta) \lambda *\left(\phi_{\varepsilon}\right)^{\gamma}(\xi+\eta) m(\xi, \eta) d \xi d \eta \\
=C \sum_{t \in \mathbb{R}} \sum_{s \in \mathbb{R}} m(t, s) \mu(\{t\}) \nu(\{s\}) \lambda(\{(t+s)\})
\end{gathered}
$$

for all measures $\mu, \nu, \lambda$ having their supports on finite sets of points.

On the other hand, from (i) and Lemma 2.1, we have

$$
\begin{gathered}
\left|\int_{\mathbb{R}^{2}} \mu *\left(\phi_{\varepsilon}\right)^{\alpha}(\xi) \nu *\left(\phi_{\varepsilon}\right)^{\beta}(\eta) \lambda *\left(\phi_{\varepsilon}\right)^{\gamma}(\xi+\eta) m(\xi, \eta) d \xi d \eta\right| \\
\leq K\left\|\widehat{\mu} \widehat{\left(\phi_{\varepsilon}\right)^{\alpha}}\right\|_{p_{1}}\left\|\hat{\nu} \widehat{\left(\phi_{\varepsilon}\right)^{\beta}}\right\|_{p_{2}}\left\|\hat{\lambda} \widehat{\left(\phi_{\varepsilon}\right)^{\gamma}}\right\|_{p_{3}^{\prime}} .
\end{gathered}
$$

We now choose $\alpha=1 / p_{1}^{\prime}, \beta=1 / p_{2}^{\prime}$, and $\gamma=1 / p_{3}$. Since $\left(\phi_{\varepsilon}\right)^{\alpha}=\varepsilon^{1-\alpha} / \alpha^{1 / 2} \phi_{\varepsilon \alpha^{-1 / 2}}$, we get $\widehat{\left(\phi_{\varepsilon}\right)^{\alpha}}(\xi)=C_{\alpha} \varepsilon^{1 / p_{1}} e^{-\varepsilon^{2} \xi^{2} / 2 \alpha}, \widehat{\left(\phi_{\varepsilon}\right)^{\beta}}(\xi)=C_{\beta} \varepsilon^{1 / p_{2}} e^{-\varepsilon^{2} \xi^{2} / 2 \beta}$, and $\widehat{\left(\phi_{\varepsilon}\right)^{\gamma}}(\xi)=C_{\gamma} \varepsilon^{1 / p_{3}} e^{-\varepsilon^{2} \xi^{2} / 2 \gamma}$ for some constants $C_{\alpha}, C_{\beta}$, and $C_{\gamma}$.

Now taking into account that $\int_{\mathbb{R}} e^{-\varepsilon^{2} p_{1} \xi^{2} / 2 \alpha} d \xi=C_{\alpha}^{\prime} \varepsilon^{-1}$, we have

$$
\left\|\widehat{\mu} \widehat{\left(\phi_{\varepsilon}\right)^{\alpha}}\right\|_{p_{1}}=C\left(\frac{1}{A(\varepsilon)} \int_{\mathbb{R}}|\widehat{\mu}(\xi)|^{p_{1}} \varepsilon^{-p_{1} \varepsilon^{2} \xi^{2} / 2 \alpha} d \xi\right)^{1 / p_{1}},
$$

for $A(\varepsilon)=\int_{\mathbb{R}} e^{-\varepsilon^{2} p_{1} \xi^{2} / 2 \alpha} d \xi$. Hence $C\|\hat{\mu}\|_{B_{p_{1}}}=\lim _{\varepsilon \rightarrow 0}\left\|\hat{\mu} \hat{\phi}_{\varepsilon}^{\alpha}\right\|_{p_{1}}$.

Applying a similar procedure for $\nu$ and $\lambda$, we finish this implication. 
(ii) $\Rightarrow$ (i). From (ii) we can get that the inequality holds for all finite measures $\mu, v, \lambda$, with countable supports. We take $\phi, \psi$, and $\rho$ such that $\hat{\phi}, \hat{\psi}$, and $\hat{\rho}$ have compact support contained in $[-N / 2, N / 2]$ for $N$ big enough. Now consider $\mu_{N}, \nu_{N}$, and $\lambda_{N}$ the measures with support in $(1 / N) \mathbb{Z}$ whose Fourier transform coincides with the periodic extensions of $\hat{\phi}, \hat{\psi}$, and $\hat{\rho}$. In particular, we have

$$
\mu_{N}\left(\left\{\frac{n}{N}\right\}\right)=\frac{1}{N} \phi\left(\frac{n}{N}\right), \quad v_{N}\left(\left\{\frac{n}{N}\right\}\right)=\frac{1}{N} \psi\left(\frac{n}{N}\right), \quad \lambda_{N}\left(\left\{\frac{n}{N}\right\}\right)=\frac{1}{N} \rho\left(\frac{n}{N}\right) .
$$

Therefore we have

$$
\begin{gathered}
\lim _{N \rightarrow \infty} N \sum_{(t, s) \in \mathbb{R} \times \mathbb{R}} m(t, s) \mu_{N}(\{t\}) \nu_{N}(\{s\}) \lambda_{N}(\{t+s\}) \\
\quad=\lim _{N \rightarrow \infty} \sum_{(n, m) \in \mathbb{Z} \times \mathbb{Z}} m\left(\frac{n}{N}, \frac{m}{N}\right) \phi\left(\frac{n}{N}\right) \psi\left(\frac{m}{N}\right) \rho\left(\frac{n+m}{N}\right) \frac{1}{N^{2}} \\
=\int_{\mathbb{R}^{2}} m(\xi, \nu) \phi(\xi) \psi(\eta) \rho(\xi+\eta) d \xi d \eta .
\end{gathered}
$$

Now observe that $\left\|\hat{\mu}_{N}\right\|_{B_{p_{1}}}=\left((1 / 2 N) \int_{-N}^{N}|\hat{\phi}(\xi)|^{p_{1}} d \xi\right)^{1 / p_{1}}=(1 / 2 N)^{1 / p_{1}}\|\hat{\phi}\|_{p_{1}}$ and the same for the others.

Using that $\left\|\hat{\mu}_{N}\right\|_{B_{p_{1}}} \cdot\left\|\hat{\nu}_{N}\right\|_{B_{p_{2}}}\left\|\hat{\lambda}_{N}\right\|_{B_{p_{3}^{\prime}}}=1 / 2 N$ and passing to the limit, we get the result.

Remark 2.5. We point out that condition (ii) in Theorem 2.4 is simply a way to say that $m$ defines a multiplier on $\mathbb{D} \times \mathbb{D}$ where $\mathbb{D}$ is the group $\mathbb{R}$ with the discrete topology (see [6]).

Recall that a function $m$ is called regulated if

$$
\lim _{\varepsilon \rightarrow 0} \frac{1}{4 \varepsilon^{2}} \int_{-\varepsilon}^{\varepsilon} \int_{-\varepsilon}^{\varepsilon} m(x-s, y-t) d s d t=m(x, y)
$$

for all $(x, y) \in \mathbb{R}^{2}$.

Theorem 2.6. Let $m(\xi, \eta)$ be a bounded regulated function on $\mathbb{R} \times \mathbb{R}$. Then $m$ is a $\left(p_{1}, p_{2}\right)$ multiplier on $\mathbb{R} \times \mathbb{R}$ if and only if there exists a constant $K$ so that

$$
\left|\sum_{t \in \mathbb{R}} \sum_{s \in \mathbb{R}} m(t, s) \mu(\{t\}) \nu(\{s\}) \lambda(\{t+s\})\right| \leq K\|\hat{\mu}\|_{B_{p_{1}}}\|\hat{\nu}\|_{B_{p_{2}}}\|\hat{\lambda}\|_{B_{p_{3}^{\prime}}}
$$

for all measures $\mu, \nu, \lambda$ having their supports on finite sets of points.

Proof. Assume that $m$ is a $\left(p_{1}, p_{2}\right)$-multiplier. Let $\Phi(s, t)=(1 / 4) \chi_{[-1,1]}(s) \chi_{[-1,1]}(t)$ and $\Phi_{\varepsilon}(\xi, \eta)=\left(1 / \varepsilon^{2}\right) \Phi(\xi / \varepsilon, \eta / \varepsilon)$ for $\varepsilon>0$. Now Lemma 2.2, Theorem 2.4, and the fact that $m(x, y)=\lim _{\varepsilon \rightarrow 0} m * \Phi_{\varepsilon}(x, y)$ give the direct implication. 
Conversely, assume (2.16) for $\mu, \nu, \lambda$ having finite supports. Then

$$
\begin{aligned}
\sum_{t \in \mathbb{R}} \sum_{s \in \mathbb{R}}\left(m * \Phi_{\varepsilon}\right)(t, s) \mu(\{t\}) \nu(\{s\}) \lambda(\{t+s\}) \\
=\int_{\mathbb{R}^{2}}\left(\sum_{t \in \mathbb{R}} \sum_{s \in \mathbb{R}} m(t-u, s-v) \mu(\{t\}) \nu(\{s\}) \lambda(\{t+s\})\right) \Phi_{\varepsilon}(u, v) d u d v \\
=\int_{\mathbb{R}^{2}}\left(\sum_{t \in \mathbb{R}} \sum_{s \in \mathbb{R}} m(t, s) \mu(\{t+u\}) \nu(\{s+v\}) \lambda(\{t+s+u+v\})\right) \Phi_{\varepsilon}(u, v) d u d v
\end{aligned}
$$

This shows that $m * \Phi_{\varepsilon}$ verifies (2.16) with a uniform constant for all $\varepsilon>0$. Now apply Theorem 2.4 to get that $m * \Phi_{\varepsilon}$ are $\left(p_{1}, p_{2}\right)$-multipliers with uniform norm.

Finally we have that for $\phi, \psi, \nu \in \mathscr{Y}$,

$$
\begin{aligned}
& \left|\int_{\mathbb{R}^{2}} \phi(\xi) \psi(\eta) \nu(\xi+\eta) m(\xi, \eta) d \xi d \eta\right| \\
& \quad=\left|\lim _{\varepsilon \rightarrow 0} \int_{\mathbb{R}^{2}} \phi(\xi) \psi(\eta) \nu(\xi+\eta)\left(m * \Phi_{\varepsilon}\right)(\xi, \eta) d \xi d \eta\right| \\
& \quad \leq C\|\hat{\phi}\|_{p_{1}}\|\hat{\psi}\|_{p_{2}}\|\hat{\nu}\|_{p_{3}^{\prime} .}
\end{aligned}
$$

The result now follows from Lemma 2.1.

\section{Transference theorems}

We mention the formulations for $\left(p_{1}, p_{2}\right)$-multipliers on the groups $\mathbb{T}$ and $\mathbb{Z}$ which follow directly from duality.

Lemma 3.1. Let $\tilde{m}(t, s)$ be a bounded measurable function on $\mathbb{T} \times \mathbb{T}$. Then $m$ is a $\left(p_{1}, p_{2}\right)$ multiplier on $\mathbb{T} \times \mathbb{T}$ if and only if there exists a constant $K$ so that

$$
\left|\int_{-1 / 2}^{1 / 2} \int_{-1 / 2}^{1 / 2} P_{a}(t) P_{b}(s) P_{c}(t+s) \tilde{m}(t, s) d t d s\right| \leq K\|a\|_{p_{1}}\|b\|_{p_{2}}\|c\|_{p_{3}^{\prime}}
$$

for all finite sequences $(a(n))_{n},(b(n))_{n},(c(n))_{n}$, where $P_{a}(t)=\sum_{n} a(n) e^{2 \pi i n t}$.

Lemma 3.2. Let $\left(m_{k, k^{\prime}}\right)$ be a bounded sequence on $\mathbb{Z} \times \mathbb{Z}$. Then $m$ is a $\left(p_{1}, p_{2}\right)$-multiplier on $\mathbb{Z} \times \mathbb{Z}$ if and only if there exists a constant $K$ so that

$$
\left|\sum_{k \in \mathbb{Z}} \sum_{k^{\prime} \in \mathbb{Z}} m_{k, k^{\prime}} \hat{P}(k) \hat{Q}\left(k^{\prime}\right) \hat{R}\left(k+k^{\prime}\right)\right| \leq K\|P\|_{p_{1}}\|Q\|_{p_{2}}\|R\|_{p_{3}^{\prime}}
$$

for all trigonometric polynomials $P, Q$, and $R$.

Theorem 3.3 (see $[7$, Theorem 1$]$ ). Let $m(\xi, \eta)$ be a regulated bounded function on $\mathbb{R} \times \mathbb{R}$. If $m(\xi, \eta)$ is a $\left(p_{1}, p_{2}\right)$-multiplier on $\mathbb{R} \times \mathbb{R}$, then $\left(m\left(k, k^{\prime}\right)\right)_{k, k^{\prime}}$ is a $\left(p_{1}, p_{2}\right)$-multiplier on $\mathbb{Z} \times \mathbb{Z}$. 
Proof. According to Lemma 3.2, we have to show that there exists a constant $K$ so that

$$
\left|\sum_{k \in \mathbb{Z}} \sum_{k^{\prime} \in \mathbb{Z}} m\left(k, k^{\prime}\right) \hat{P}(k) \hat{Q}\left(k^{\prime}\right) \hat{R}\left(k+k^{\prime}\right)\right| \leq K\|P\|_{p_{1}}\|Q\|_{p_{2}}\|R\|_{p_{3}^{\prime}}
$$

for all trigonometric polynomials $P, Q$, and $R$.

This follows by selecting the measures $\mu, v, \lambda$ in Theorem 2.6 such that $\hat{\mu}=P, \hat{v}=Q$, and $\hat{\lambda}=R$.

Theorem 3.4. Let $m(\xi, \eta)$ be a bounded regulated function on $\mathbb{R} \times \mathbb{R}$. The following are equivalent:

(i) $m(\xi, \eta)$ is a $\left(p_{1}, p_{2}\right)$-multiplier on $\mathbb{R} \times \mathbb{R}$;

(ii) $m\left(\varepsilon^{\cdot}, \varepsilon^{\cdot}\right) \chi_{[-1 / 2 \varepsilon, 1 / 2 \varepsilon]} \chi_{[-1 / 2 \varepsilon, 1 / 2 \varepsilon]}$ (extended by periodicity) are uniformly bounded $\left(p_{1}, p_{2}\right)$-multipliers on $\mathbb{T} \times \mathbb{T}$.

Proof. (i) $\Rightarrow$ (ii). Using Lemma 3.1, it suffices to show that for any finite sequences $(a(n))_{n}$, $(b(n))_{n}$, and $(c(n))_{n}$ with $\|a\|_{p_{1}}=\|b\|_{p_{2}}=\|c\|_{p_{3}^{\prime}}=1$, there exists a constant $K>0$ such that

$$
\left|\int_{-1 / 2}^{1 / 2} \int_{-1 / 2}^{1 / 2} m(\xi, \eta) P_{a}(\xi) P_{b}(\eta) P_{c}(\xi+\eta) d \xi d \eta\right| \leq K,
$$

where $P_{a}(\xi)=\sum_{n} a(n) e^{2 \pi i n \xi}$.

Since $P_{a}(x) \chi_{[-1 / 2,1 / 2]}(x)=\hat{\phi}_{a}(x)$, where $\phi_{a}(x)=\sum_{n} a(n)(\sin (\pi(x-n)) / \pi(x-n))$, and $P_{c}(x) \chi_{[-1,1]}(x)=\hat{\psi}_{c}(x)$, where $\psi_{c}(x)=\sum_{n} c(n)(\sin (2 \pi(x-n)) / \pi(x-n))$, we can write

$$
\begin{array}{rl}
\int_{-1 / 2}^{1 / 2} \int_{-1 / 2}^{1 / 2} & m(\xi, \eta) P_{a}(\xi) P_{b}(\eta) P_{c}(\xi+\eta) d \xi d \eta \\
= & \int_{\mathbb{R}} \int_{\mathbb{R}} m(\xi, \eta) \hat{\phi}_{a}(\xi) \hat{\phi}_{b}(\eta) \hat{\psi}_{c}(\xi+\eta) d \xi d \eta .
\end{array}
$$

Using now the assumption and Shanon's sampling theorem, one gets $\left\|\psi_{a}\right\|_{L^{p}(\mathbb{R})} \leq$ $C_{1}\left\|\phi_{a}\right\|_{L^{p}(\mathbb{R})} \leq C_{2}\|a\|_{\ell_{p}} \leq C_{3}\left\|\psi_{a}\right\|_{L^{p}(\mathbb{R})}$ for some constants $C_{i}$ for $i=1,2,3$. Hence the desired inequality follows.

Now we apply Lemma 2.3 to get the result for each $\varepsilon$.

(ii) $\Rightarrow($ i). We take $\phi$ and $\psi$ such that $\operatorname{supp} \phi$ and $\operatorname{supp} \psi$ are contained in $[-1 / 4,1 / 4]$. For a fixed $u \in[-1 / 2,1 / 2]$, consider the periodic extensions of the functions $\hat{\phi}(\xi) e^{2 \pi i u \xi}$, $\widehat{\psi}(\eta) e^{2 \pi i u \eta}$ to be denoted $\widetilde{P}_{u}$ and $\widetilde{Q}_{u}$, respectively.

If $a^{u}(n)=\int_{-1 / 2}^{1 / 2} \widetilde{P}_{u}(\xi) e^{-i 2 \pi n \xi} d \xi, b^{u}(n)=\int_{-1 / 2}^{1 / 2} \widetilde{Q}_{u}(\xi) e^{-i 2 \pi n \xi} d \xi$ for all $n \in \mathbb{Z}$, we have that if $x=k+u$ for some $k \in \mathbb{Z}$ and $u \in[-1 / 2,1 / 2)$,

$$
\begin{array}{rl}
\int_{\mathbb{R}} \int_{\mathbb{R}} & m(\xi, \eta) \hat{\phi}(\xi) \hat{\psi}(\eta) e^{2 \pi i x(\xi+\eta)} d \xi d \eta \\
\quad=\int_{-1 / 2}^{1 / 2} \int_{-1 / 2}^{1 / 2} m(\xi, \eta) \widetilde{P}_{u}(\xi) \widetilde{Q}_{u}(\eta) e^{2 \pi i k(\xi+\eta)} d \xi d \eta .
\end{array}
$$

Let $\tilde{m}(\xi, \eta)=m(\xi, \eta) \chi_{[-1 / 2,1 / 2]}(\xi) \chi_{[-1 / 2,1 / 2]}(\eta)$. Hence for $x=u+k$,

$$
\mathscr{C}_{m}(\phi, \psi)(x)=\mathscr{D}_{\tilde{m}}\left(a^{u}, b^{u}\right)(k) .
$$


Now

$$
\begin{aligned}
\int_{\mathbb{R}}\left|\mathscr{C}_{m}(\phi, \psi)(x)\right|^{p_{3}} d x & \\
& =\sum_{k} \int_{-1 / 2}^{1 / 2}\left|\mathscr{C}_{m}(\phi, \psi)(k+u)\right|^{p_{3}} d u \\
& =\int_{-1 / 2}^{1 / 2} \sum_{k}\left|\mathscr{D}_{\tilde{m}}\left(a^{u}, b^{u}\right)(k)\right|^{p_{3}} d u \\
& \leq\left\|\mathscr{D}_{\tilde{m}}\right\|^{p_{3}} \int_{-1 / 2}^{1 / 2}\left(\sum_{k}\left|a^{u}(k)\right|^{p_{1}}\right)^{p_{3} / p_{1}}\left(\sum_{k}\left|b^{u}(k)\right|^{p_{2}}\right)^{p_{3} / p_{2}} d u \\
& \leq\left\|\mathscr{D}_{\tilde{m}}\right\|^{p_{3}}\left(\int_{-1 / 2}^{1 / 2} \sum_{k}\left|a^{u}(k)\right|^{p_{1}} d u\right)^{p_{3} / p_{1}}\left(\int_{-1 / 2}^{1 / 2} \sum_{k}\left|b^{u}(k)\right|^{p_{2}} d u\right)^{p_{3} / p_{2}} \\
& =\left\|\mathscr{D}_{\tilde{m}}\right\|^{p_{3}}\left(\int_{-1 / 2}^{1 / 2} \sum_{k}|\phi(u+k)|^{p_{1}} d u\right)^{p_{3} / p_{1}}\left(\int_{-1 / 2}^{1 / 2} \sum_{k}|\psi(u+k)|^{p_{2}} d u\right)^{p_{3} / p_{2}} \\
& =\left\|\mathscr{D}_{\tilde{m}}\right\|^{p_{3}}\|\phi\|_{p_{1}}^{p_{3}}\|\psi\|_{p_{2}}^{p_{3}} .
\end{aligned}
$$

In the general case if $\phi, \psi$ are such that $\hat{\phi}, \hat{\psi}$ have compact support, then there exists $\varepsilon>0$ so that $\hat{\phi}_{\varepsilon}, \hat{\psi}_{\varepsilon}$ have their support in $[-1 / 4,1 / 4]$. Now observe that

$$
\mathscr{C}_{m}(\phi, \psi)(x)=\varepsilon^{2} C_{m(\varepsilon \cdot \varepsilon \cdot)}\left(\phi_{\varepsilon}, \psi_{\varepsilon}\right)(\varepsilon x) .
$$

Applying the previous case and the assumption, we obtain

$$
\begin{aligned}
\left\|\mathscr{C}_{m}(\phi, \psi)\right\|_{p_{3}} & =\varepsilon^{2-1 / p_{3}}\left\|C_{m(\varepsilon, \varepsilon \cdot)}\left(\phi_{\varepsilon}, \psi_{\varepsilon}\right)\right\|_{p_{3}} \\
& \leq K \varepsilon^{2-1 / p_{3}}\left\|\phi_{\varepsilon}\right\|_{p_{1}}\left\|\psi_{\varepsilon}\right\|_{p_{2}} \\
& =K \varepsilon^{2-1 / p_{3}}\|\phi\|_{p_{1}} \varepsilon^{-1 / p_{1}^{\prime}}\|\psi\|_{p_{1}} \varepsilon^{-1 / p_{2}^{\prime}} \\
& =K\|\phi\|_{p_{1}}\|\psi\|_{p_{1}} .
\end{aligned}
$$

\section{Acknowledgment}

The author is partially supported by Grants no. PB98-0146 and no. BFM2002-04013.

\section{References}

[1] P. Auscher and M. J. Carro, On relations between operators on $\mathbf{R}^{N}, \mathbf{T}^{N}$ and $\mathbf{Z}^{N}$, Studia Math. 101 (1992), no. 2, 165-182.

[2] O. Blasco and F. Villarroya, Transference of bilinear multiplier operators on Lorentz spaces, Illinois J. Math. 47 (2003), no. 4, 1327-1343.

[3] R. R. Coifman and Y. Meyer, Commutateurs d'intégrales singulières et opérateurs multilinéaires, Ann. Inst. Fourier (Grenoble) 28 (1978), no. 3, xi, 177-202 (French).

[4] - Fourier analysis of multilinear convolutions, Calderón's theorem, and analysis of Lipschitz curves, Euclidean Harmonic Analysis (Proc. Sem., Univ. Maryland, College Park, Md, 1979), Lecture Notes in Math., vol. 779, Springer, Berlin, 1980, pp. 104-122. 


\section{Bilinear multipliers and transference}

[5] R. R. Coifman and G. Weiss, Transference Methods in Analysis, Memoirs of the American Mathematical Society, vol. 31, American Mathematical Society, Rhode Island, 1977.

[6] K. de Leeuw, On L multipliers, Ann. of Math. (2) 81 (1965), 364-379.

[7] D. Fan and S. Sato, Transference on certain multilinear multiplier operators, J. Aust. Math. Soc. 70 (2001), no. 1, 37-55.

[8] J. E. Gilbert and A. R. Nahmod, Boundedness of bilinear operators with nonsmooth symbols, Math. Res. Lett. 7 (2000), no. 5-6, 767-778.

[9] , Bilinear operators with non-smooth symbol. I, J. Fourier Anal. Appl. 7 (2001), no. 5, $435-467$.

[10],$L^{p}$-boundedness for time-frequency paraproducts. II, J. Fourier Anal. Appl. 8 (2002), no. 2, 109-172.

[11] L. Grafakos and N. J. Kalton, The Marcinkiewicz multiplier condition for bilinear operators, Studia Math. 146 (2001), no. 2, 115-156.

[12] L. Grafakos and R. H. Torres, Multilinear Calderón-Zygmund theory, Adv. Math. 165 (2002), no. $1,124-164$.

[13] L. Grafakos and G. Weiss, Transference of multilinear operators, Illinois J. Math. 40 (1996), no. 2, 344-351.

[14] E. Hewitt and K. A. Ross, Abstract Harmonic Analysis. Vol. II: Structure and Analysis for Compact Groups. Analysis on Locally Compact Abelian Groups, Die Grundlehren der mathematischen Wissenschaften, vol. 152, Springer, Berlin, 1970.

[15] C. E. Kenig and E. M. Stein, Multilinear estimates and fractional integration, Math. Res. Lett. 6 (1999), no. 1, 1-15.

[16] M. Lacey and C. Thiele, $L^{p}$ estimates on the bilinear Hilbert transform for $2<p<\infty$, Ann. of Math. (2) 146 (1997), no. 3, 693-724.

[17] - On Calderón's conjecture, Ann. of Math. (2) 149 (1999), no. 2, 475-496.

[18] M. T. Lacey, On the bilinear Hilbert transform, Doc. Math. (1998), no. Extra Vol. II, 647-656.

[19] Y. Meyer and R. R. Coifman, Ondelettes et Opérateurs. III. Opérateurs Multilinéaires. [Wavelets and Operators. III. Multilinear Operators], Actualités Mathématiques, Hermann, Paris, 1991.

[20] C. Muscalu, T. Tao, and C. Thiele, Multi-linear operators given by singular multipliers, J. Amer. Math. Soc. 15 (2002), no. 2, 469-496.

[21] E. M. Stein and G. Weiss, Introduction to Fourier Analysis on Euclidean Spaces, Princeton Mathematical Series, no. 32, Princeton University Press, New Jersey, 1971.

Oscar Blasco: Departamento de Análisis Matemático , Universitat de València, 46100-Burjassot, València, Spain

E-mail address: oscar.blasco@uv.es 


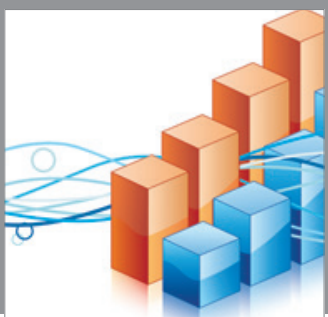

Advances in

Operations Research

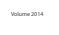

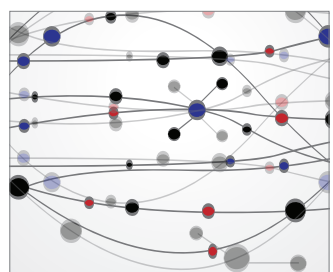

\section{The Scientific} World Journal
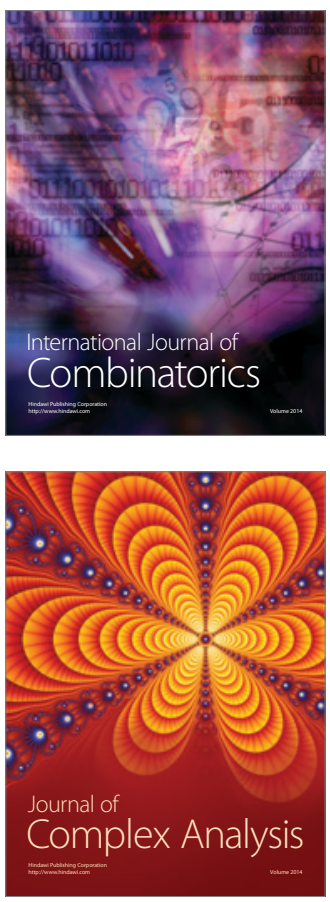

International Journal of

Mathematics and

Mathematical

Sciences
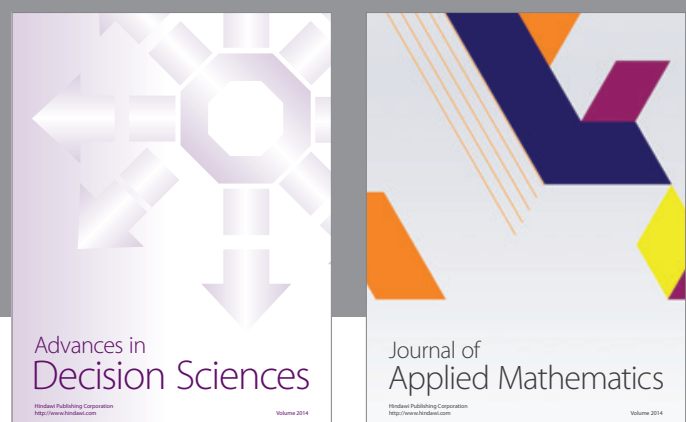

Journal of

Applied Mathematics
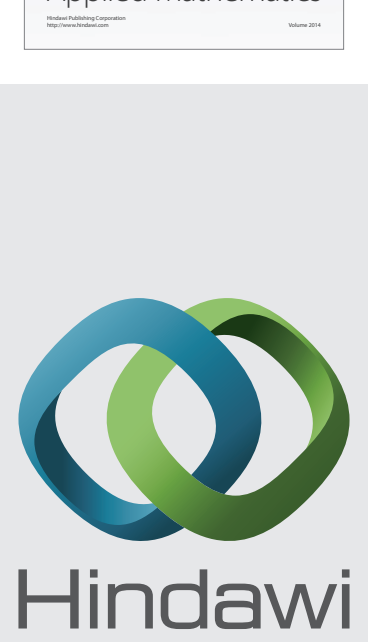

Submit your manuscripts at http://www.hindawi.com
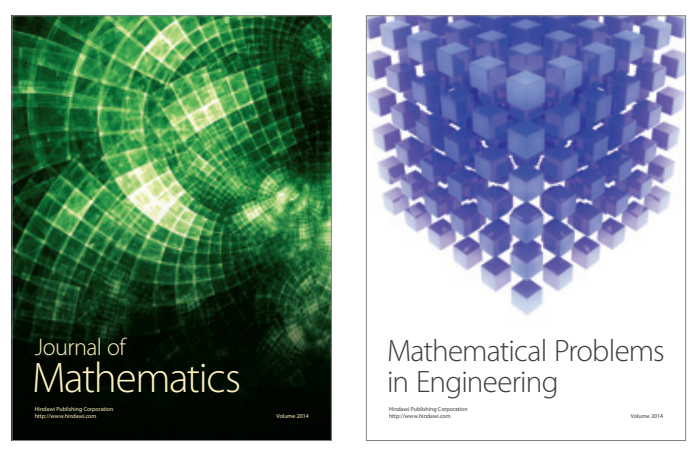

Mathematical Problems in Engineering
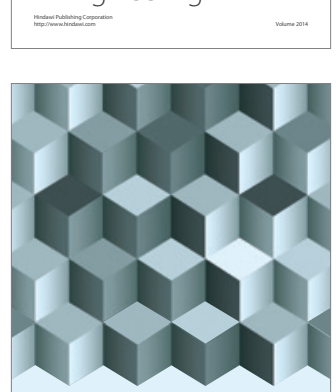

Journal of

Function Spaces
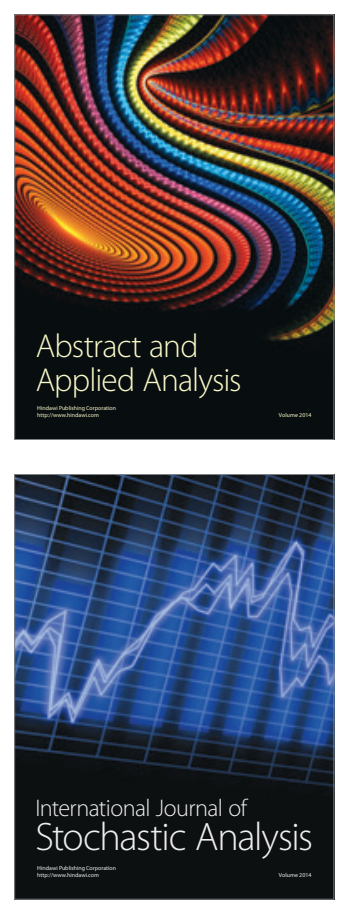

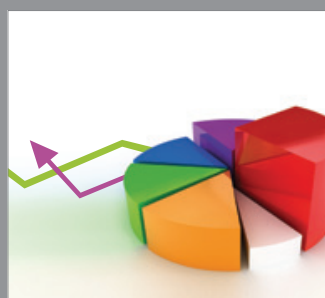

ournal of

Probability and Statistics

Promensencen
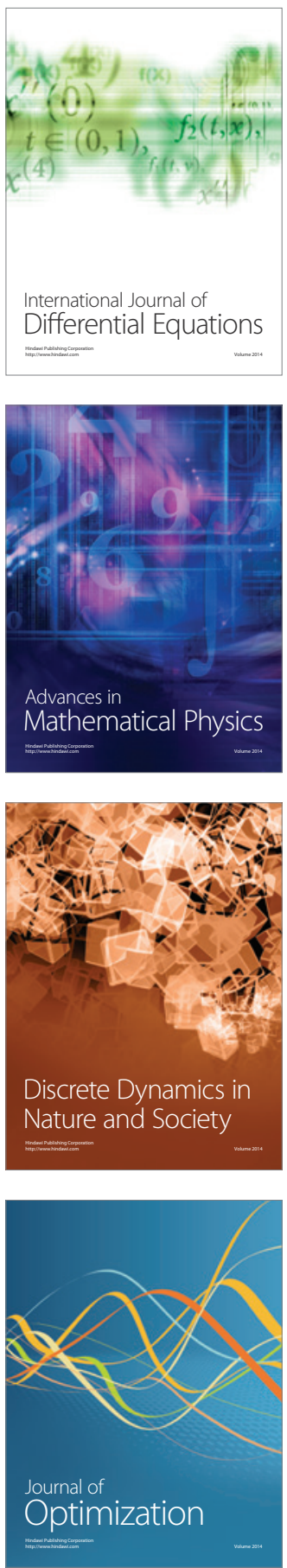